\title{
COLLES'S CLASSIC DESCRIPTION OF FRACTURES OF THE LOWER END OF THE RADIUS*
}

On the Fracture of the Carpal extremity of the Radius. By A. Colles, M. D., one of the Professors of Anatomy and Surgery in the Royal College of Surgeons in Ireland.

THE injury to which I wish to direct the attention of surgeons, has not, as far as I know, been described by any author; indeed the form of the carpal extremity of the radius would rather incline us to question its being liable to fracture. The absence of crepitus, and of the other common symptoms of fracture, together with the swelling which instantly arises in this, as in other injuries of the wrist, render the difficulty of ascertaining the real nature of the case very considerable.

This fracture takes place at about an inch and a half above the carpal extremity. of the radius, and exhibits the following appearances.

The posterior surface of the limb presents a considerable deformity; for a depression is seen in the fore-arm, about an inch and a half above the end of this bone, while a considerable swelling occupies the wrist and metacarpus. Indeed, the carpus and base of metacarpus appear to be thrown backward so much, as on first view to excite a suspicion that the carpus has been dislocated forward.

On viewing the anterior surface of the limb, we observe a considerable fulness, as if caused by the flexor tendons being thrown forwards. This fulness extends upwards to about one-third of the length of the fore-arm, and terminates below at the upper edge of the annular ligament of the wrist. The extremity of the ulna is seen projecting towards the palm and inner edge of the limb; the degree, however, in which this projection takes place, is different in different instances.

If the surgeon proceed to investigate the nature of this injury, he will find that the end of the ulna admits of being readily moved backwards and forwards.

On the posterior surface, he will discover by the touch that the swelling on the wrist, and metacarpus, is not caused entirely by an effusion among the softer parts; he will perceive that the ends of the metacarpal, and second row of carpal bones, form no small part of it. This, strengthening the suspicion which the first view of the case had excited, leads him to examine, in a more particular manner, the anterior part of the joint; but the want of that solid resistance, which a dislocation of the carpus forward must occasion, forces him to abandon this notion, and leaves him in a state of perplexing uncertainty as to the real nature of the injury. He will, therefore, endeavour to gain some information, by examining the bones of the fore-arm. The facility with which, (as was before noticed,) the ulna can be moved backward and forward, does not furnish him with any useful hint. When he moves his fingers along the anterior surface of the radius, he finds it more full and prominent than is natural; a similar examination of the posterior surface of this bone, induces him to think that a depression is felt about an inch and half above its carpal extremity. He now expects to find satisfactory proofs of a fracture of the radius at this spot. For this purpose, he attempts to move the broken pieces of the bone in opposite directions; but, although the patient is by this examination subjected to considerable pain, yet, neither crepitus nor a yielding of the bone at the seat of fracture, nor any other positive evidence of the existence of such an injury is thereby obtained. The patient complains of severe pain as often as an attempt is made to give to the limb the motions of pronation and supination.

If the surgeon lock his hand in that of the patient's, and make extension, even with a moderate force, he restores the limb to its natural form, but the distortion of the limb instantly returns on the extension being removed. Should the facility with which a moderate extension restores the limb to its form, induce the practitioner to treat this as a case of sprain, he will find, after a lapse of time sufficient for the removal of similar swellings, the deformity undiminished. Or, should he mistake

* Reprinted from the Edinburgh Medical and Surgical Journal, Volume 10, p. 182 (1814). 
the case for a dislocation of the wrist, and attempt to retain the parts in situ by tight bandages and splints, the pain caused by the pressure on the back of the wrist will force him to unbind them in a few hours; and if they be applied more loosely, he will find, at the expiration of a few weeks, that the deformity still exists in its fullest extent, and that it is now no longer to be removed by making extension of the limb. By such mistakes the patient is doomed to endure for many months considerable lameness and stiffness of the limb, accompanied by severe pains on attempting to bend the hand and fingers. One consolation only remains, that the limb will at some remote period again enjoy perfect freedom in all its motions, and be completely exempt from pain; the deformity, however, will remain undiminished through life.

The unfavourable result of some of the first cases of this description which came under my care, forced me to investigate with peculiar anxiety the nature of the injury. But while the absence of crepitus and of the other usual symptoms of fracture rendered the diagnosis extremely difficult; a recollection of the superior strength and thickness of this part of the radius, joined to the mobility of its articulation with the carpus and ulna, rather inclined me to question the possibility of a fracture taking place at this part of the bone. At last, after many unsuccessful trials, I hit upon the following simple method of examination, by which I was enabled to ascertain, that the symptoms above enumerated actually arose from a fracture, seated about an inch and a half above the carpal extremity of the radius.

Let the surgeon apply the fingers of one hand to the seat of the suspected fracture, and, locking the other hand in that of the patient, make a moderate extension, until he observes the limb restored to its natural form. As soon as this is effected, let him move the patient's hand backward and forward; and he will, at every such attempt, be sensible of a yielding of the fractured ends of the bone, and this to such a degree as must remove all doubt from his mind.

The nature of this injury once ascertained, it will be a very easy matter to explain the different phenomena attendant on it, and to point out a method of treatment which will prove completely successful. The hard swelling which appears on the back of the hand, is caused by the carpal surface of the radius being directed slightly backwards instead of looking directly downwards. The carpus and metacarpus retaining their connections with this bone, must follow it in its derangements, and cause the convexity above alluded to. This change of direction in the articulating surface of the radius is caused by the tendons of the extensor muscles of the thumb, which pass along the posterior surface of the radius in sheaths firmly connected with the inferior extremity of this bone. The broken extremity of the radius being thus drawn backwards, causes the ulna to appear prominent towards the palmar surface, whlle it is possibly thrown more towards the inner or ulnar side of the limb, by the upper end of the fagment of the radius pressing against it in that direction. The separation of these two bones from each other is facilitated by a previous rupture of their capsular ligament; an event which may readily be occasioned by the violence of the injury. An effusion into the sheaths of the flexor tendons will account for that swelling which occupies the limb anteriorly.

It is obvious that, in the treatment of this fracture, our attention should be principally directed to guard against the carpal end of the radius being drawn backwards. For this purpose, while assistants hold the limb in a middle state between pronation and supination, let a thick and firm compress be applied transversely on the anterior surface of the limb, at the seat of fracture, taking care that it shall not press on the ulna; let this be bound on firmly with a roller, and then let a tin splint, formed to the shape of the arm, be applied to both its anterior and posterior surfaces. In cases where the end of the ulna has appeared much displaced, I have laid a very narrow wooden splint along the naked side of this bone. This latter splint, I now think, should be used in every instance, as, by pressing the extremity of the ulna against the side of the radius, it will tend to oppose the 
displacement of the fractured end of this bone. It is scarcely necessary to observe, that the two principal splints should be much more narrow at the wrist than those in general use, and should also extend to the roots of the fingers, spreading out so as to give a firm support to the hand. The cases treated on this plan have all recovered without the smallest defect or deformity of the limb, in the ordinary time for the cure of fractures.

I cannot conclude these observations without remarking, that were my opinion to be drawn from those cases only which have occurred to me, I should consider this as by far the most common injury to which the wrist or carpal extremities of the radius and ulna are exposed. During the last three years, I have not met with a single instance of Dessault's dislocation of the inferior end of the radius, while I have had opportunities of seeing a vast number of the fracture of the lower end of this bone.

Stephens Green, February' 21, I8I4.

\section{INSTABILITY OF THE UPPER CERVICAL SPINE}

Dislocation and subluxation of the atlanto-axial joint are being recognised with increasing frequency by radiologists, rheumatologists, anaesthetists and orthopaedic surgeons. Although atlanto-axial instability is seen with congenital absence of the dens, hypoplasia and fracture of the dens, rheumatoid arthritis is now considered to be the most common cause in adults.

The person with an unstable atlanto-axial joint is leading a rather precarious life. sometimes with a fatal outcome, as in the case reported by Beighton and Craig in which tetraplegia and death occurred in a child with the Morquio syndrome. Many patients have lived for years without neurological impairment despite an ununited fracture of the dens or instability from rheumatoid arthritis. Nevertheless, an injury superimposed on this condition may cause serious disability and death. The risk is probably greater in those with an intact dens, as in rheumatoid arthritis, than in those with an ununited fracture, for cord compression occurs more easily in the former.

Good radiological examination is essential to establish the diagnosis. This is well pointed out in the papers by Rana, Hancock, Taylor and Hill on upward translocation of the dens and on atlanto-axial subluxation in rheumatoid arthritis. In addition to tomography in flexion and extension, cineradiography is often of value in determining the direction and the amount of displacement. Because of the frequency of the condition in rheumatoid arthritis, flexion and extension views of the cervical spine should be part of the routine examination and they should be mandatory for patients who may require a general anaesthetic.

The treatment of atlanto-axial instability is dependent on the clinical findings. Nonoperative treatment by the use of protective collars or braces is usually adequate in the patients with rheumatoid arthritis, even when instability is marked. In the more active person with an ununited fracture of the dens, atlanto-axial stabilisation is advisable, even though the dens will probably remain ununited.

The indications for surgical treatment are pain, persistent or progressive neurological involvement, and vertebral artery compression.

There is still controversy about the methods of surgical stabilisation of the atlanto-axial joint. Should the occiput be included in the fusion and should the third cervical vertebra be included? There seems little doubt that for upward translocation of the dens, a bone graft to assist in maintaining distraction of the skull from the dens is advisable. With defects in the arch of the atlas, inclusion of the occiput in the fusion is necessary.

In most patients with atlanto-axial instability, from whatever cause, the so-called Gallie method described in this issue by McGraw and Rusch, and including post-operative support, has shown good results with a low incidence of non-union. Although their series is small, a large number of unreported patients from the same Centre over a period of twenty-five years 